\title{
The prognostic value of tumor length to resectable esophageal squamous cell carcinoma: a retrospective study
}

\author{
Xiangwei Zhang ${ }^{1}$, Yang Wang ${ }^{2}$, Cheng Li ${ }^{3}$, Jing Helmersson ${ }^{4}$, Yuanzhu Jiang ${ }^{1}$, Guoyuan Ma ${ }^{1}$, Guanghui \\ Wang ${ }^{1}$, Wei Dong ${ }^{1}$, Shaowei Sang ${ }^{\text {Corresp., }}{ }^{5}$, Jiajun Du ${ }^{\text {Corresp. } 1}$ \\ ${ }^{1}$ Department of Thoracic Surgery, Shandong Provincial Hospital Affiliated to Shandong University, Jinan, China \\ 2 Department of Medical Imaging, Shandong Provincial Hospital Affiliated to Shandong University, Jinan, China \\ 3 President's Office, Shandong Cancer Hospital Affiliated to Shandong University, Shandong Academy of Medical Sciences, Jinan, China \\ 4 Department of Public Health and Clinical Medicine, Epidemiology and Global Health, Umea University, Umea, Sweden \\ 5 Clinical Epidemiology Unit, Qilu Hospital of Shandong University, Jinan, China \\ Corresponding Authors: Shaowei Sang, Jiajun Du \\ Email address: sangshaowei1@163.com, dujiajun2011@126.com
}

Background. The current TNM classification system does not consider tumor length for patients with esophageal carcinoma (EC). This study explored the effect of tumor length, in addition to tumor depth and lymph node involvement, on survival in patients with esophageal squamous cell carcinoma (ESCC). Methods. A total of 498 ESCC patients who underwent surgical resection as the primary treatment were selected in the retrospective study. Pathological details were collected, which included tumor type, TNM stage, differentiation. Other collected information were: the types of esophageal resection, $A B O$ blood group, family history and demographic and lifestyle factors. A time-dependent receiver operating characteristic $(\mathrm{ROC})$ curve and a regression tree for survival were used to identify the cut-off point of tumor length, which was $3 \mathrm{~cm}$. Univariate and multivariate Cox proportional hazard regression models were used to identify the prognostic factors to ESCC. Results \& Discussion. The 1-, 3-, 5-year overall survival rates were found to be $82.5 \%, 55.6 \%$, and $35.1 \%$, respectively. Patients who had larger tumor length $(>3 \mathrm{~cm}$ ) had a higher risk for death than the rest patients. From univariate Cox proportional hazards regression model, the overall survival rate was significantly influenced by the depth of the tumor and lymph node involvement (either as dummy or continuous variables), Sex, and tumor length. Using these four variables in the multivariate Cox proportional hazard regression model, we found that the overall survival was significantly influenced by all variables except Sex. Therefore, in addition to the depth of the tumor and lymph node involvement (as either dummy or continuous variables), the tumor length is also an independent prognostic factor for ESCC. The overall survival rate was higher in a group with smaller tumor length $(\leq 3 \mathrm{~cm})$ than those patients with larger tumor length $(>3 \mathrm{~cm})$, no matter what the tumor stage was. Conclusion. The tumor length was found to be an 
important prognostic factor for ESCC patients without receiving neoadjuvant therapy. The modification of EC staging system may consider tumor length to better predict ESCC survival and identify higher risk patients for postoperative therapy. 
1 The prognostic value of tumor length to resectable esophageal squamous cell carcinoma: a

2 retrospective study

3 Xiangwei Zhang ${ }^{1}$, Yang Wang ${ }^{2}$, Cheng $\mathrm{Li}^{3}$, Jing Helmersson ${ }^{4}$, Yuanzhu Jiang ${ }^{1}$, Guoyuan $\mathrm{Ma}^{1}$,

4 Guanghui Wang ${ }^{1}$, Wei Dong ${ }^{1}$, Shaowei Sang ${ }^{5 *}$, Jiajun Du ${ }^{1 *}$

$5 \quad{ }^{1}$ Department of Thoracic Surgery, Shandong Provincial Hospital Affiliated to Shandong

6 University, 324 Jingwu Road, Jinan 250021, China

$7 \quad{ }^{2}$ Department of Medical Imaging, Shandong Provincial Hospital Affiliated to Shandong

8 University, 324 Jingwu Road, Jinan 250021, China

9 3President's Office, Shandong Cancer Hospital Affiliated to Shandong University, Shandong

10 Academy of Medical Sciences, Jinan, 250117, China

$11{ }^{4}$ Department of Public Health and Clinical Medicine, Epidemiology and Global Health, Umea University,

12 Umea, SE90185, Sweden

$13{ }^{5}$ Clinical Epidemiology Unit, Qilu Hospital of Shandong University, Jinan, 250012, China

$15 *$ Corresponding author:

16 Jiajun Du, E-mail: dujiajun2011@126.com

17 Tel: +86-531-6877-7837; fax: +86-531-6877-7837

18 Shaowei Sang, E-mail: sangshaowei1@163.com

20 Abstract

21 Background. The current TNM classification system does not consider tumor length for patients 
with esophageal carcinoma (EC). This study explored the effect of tumor length, in addition to tumor depth and lymph node involvement, on survival in patients with esophageal squamous cell carcinoma (ESCC).

Methods. A total of 498 ESCC patients who underwent surgical resection as the primary treatment were selected in the retrospective study. Pathological details were collected, which included tumor type, TNM stage, differentiation. Other collected information were: the types of esophageal resection, $\mathrm{ABO}$ blood group, family history and demographic and lifestyle factors. A time-dependent receiver operating characteristic (ROC) curve and a regression tree for survival were used to identify the cut-off point of tumor length, which was $3 \mathrm{~cm}$. Univariate and multivariate Cox proportional hazard regression models were used to identify the prognostic factors to ESCC.

Results \& Discussion. The 1-, 3-, 5-year overall survival rates were found to be $82.5 \%, 55.6 \%$, and $35.1 \%$, respectively. Patients who had larger tumor length $(>3 \mathrm{~cm})$ had a higher risk for death than the rest patients. From univariate Cox proportional hazards regression model, the overall survival rate was significantly influenced by the depth of the tumor and lymph node involvement (either as dummy or continuous variables), Sex, and tumor length. Using these four variables in the multivariate Cox proportional hazard regression model, we found that the overall survival was significantly influenced by all variables except Sex. Therefore, in addition to the depth of the tumor and lymph node involvement (as either dummy or continuous variables), the tumor length is also an independent prognostic factor for ESCC. The overall survival rate was higher in a group with smaller tumor length $(\leq 3 \mathrm{~cm})$ than those patients with larger tumor length 
$43(>3 \mathrm{~cm})$, no matter what the tumor stage was.

44 Conclusion. The tumor length was found to be an important prognostic factor for ESCC patients

45 without receiving neoadjuvant therapy. The modification of EC staging system may consider

46 tumor length to better predict ESCC survival and identify higher risk patients for postoperative

47 therapy.

48

49 
Introduction

51 Esophageal cancer is the eighth most common cancer worldwide and the sixth most common

52 cause of death from cancer (Ferlay et al. 2010). Esophageal adenocarcinoma (EAC) and squamous cell carcinoma (ESCC) are the predominant histological types. In the western countries, the majority of the esophageal cancers were EAC, while in the eastern countries, ESCC were predominant.

In the current American Joint Committee on Cancer (AJCC) staging system for esophageal 
71 great disease burden.

72 More accurate methods of determining prognosis in patients with esophageal cancer are needed.

73 It will benefit patients with specific and appropriate treatment options. The purpose of this study

74 is to explore the prognostic value of pathological tumor length in the context of other prognostic factors and obtain the optimum cut-off point for tumor length.

Materials \& Methods

Patients

From August 2007 to July 2014, 552 patients were identified to have undergone surgical

resection of esophageal tumor with curative intent at Shandong Provincial Hospital affiliated to

Shandong University. The patients who had histology other than squamous cell carcinoma were

excluded from this study $(\mathrm{n}=33)$. The patients who received neoadjuvant radiotherapy or

chemotherapy and noncurative resection (tumor-free margin) were also excluded from this study

$(n=21)$. A total of 498 patients with ESCC were included for analyses. The preoperative

evaluation was performed based on a complete medical history, physical examination,

endoscopic ultrasound and thoracoabdominal computed tomography scanning. Either the left or

the right thoracotomy was included in this study that mainly depended on the tumor location and

possible mediastinal lymph nodes involvement. The surgeons taking into account the

preoperative evaluation decided the extent of the esophageal resection and lymph nodes

dissection during the operation. Then the specimens were sent for pathology examination after

preservation in $10 \%$ formalin. The tumor length, differentiation, $\mathrm{T}$ classification, number of 
92

positive and removed lymph nodes were recorded according to the pathology reports. The TNM staging was performed according to the AJCC 7th edition guidelines. Other collected information were: the types of esophageal resection, $\mathrm{ABO}$ blood group, family history and demographic and lifestyle factors. After surgery, all the patients were followed up regularly by telephone interviews. Complete follow-up information for all patients until death or July 2014 was available. All subjects gave written informed consent to the study protocol, which was approved by the Ethical Committees of Shandong Provincial Hospital affiliated to Shandong University (No. 2015-055). The study was carried out in accordance with the approved guidelines.

\section{Statistical analyses}

Two methods were conducted to identify the optimum cut-off point for pathologically measured tumor length. First, we used a time-dependent receiver operator characteristic (ROC) curve to evaluate the tumor length in order to predict ESCC survival. Using the ROC curve, we identified the sensitivity and specificity at each tumor length point and the optimal tumor length cut-off point. This was found to be $3.1 \mathrm{~cm}$ (Figure S1A) - based on the highest Youden index (sensitivity + specificity -1) (Heagerty et al. 2000). Then, we used a regression tree survival analyses for pathologically measured tumor length (Gaur et al. 2011) and found it to be $2.9 \mathrm{~cm}$ (Figure S1B). The mean of these two results $-3.0 \mathrm{~cm}$ - was used as the threshold value for optimal cut-off. Based on this value, patients were divided into two groups: one with tumor length $\leq 3 \mathrm{~cm}$ and the other with tumor length $>3 \mathrm{~cm}$. Overall survival rate was estimated by the 
113 Kaplan-Meier method using the date of esophageal resection as the starting point and the date of

114 death or last follow-up as the endpoint. The Kaplan-Meier survival curves between two tumor

115 length groups were compared using the log-rank test. The association between risk factors and

116 survival was performed using a univariate Cox proportional hazards (Coxph) regression model.

117 Hazard ratios (HRs) with 95\% confidence intervals (CIs) were used to quantify the strength of

118 the association. Risk factors with $p$-values $\leq 0.1$ in univariate analyses were entered into a

119 multivariate Coxph regression model. The results of the multivariate Coxph regression model

120 were visualized in graphs. Tests and graphical diagnostics for proportional hazards were based

121 on the scaled Schoenfeld residuals, which calculated tests of the proportional hazards assumption

122 for each covariate by correlating the corresponding set of scaled Schoenfeld residuals with a

123 suitable transformation of time (Hess 1995). All statistical calculations were conducted using R

124 software (version 3.2.3) (Team 2014). Different packages were used for analysis: survivalROC

125 package in time-dependent ROC curve, rpart.plot and survival packages in regression tree for

126 survival, survival package in overall survival rate estimated by Kaplan-Meier method and the

127 log-rank test, univariate and multivariate Coxph regression analyses, tests and graphical

128 diagnostics for proportional hazards.

\section{Results}

131 The characteristics of the 498 patients are summarized in Table 1. The median follow-up time for

132 all patients was 47.2 months (IQR: 6.5-64.5 months). The median age was 59 years (range 38-81

133 years); majority of patients were male (78.9\%). Over $50 \%$ of the patients had over 10 years of 
134 smoking or alcohol intake. Over $80 \%$ patients' relatives (first-degree) had history of cancer,

135 which includes oral, esophagus, lung, stomach, nasopharynx, liver, pancreatic, rectal, prostate

136 cancer, etc. Majority patients (63\%) had tumor with a length larger than $3 \mathrm{~cm}$.

137 Figure 1 showed the Kaplan-Meier survival curves. The 1-, 3-, 5-year OS rates were 82.5\%,

$13855.6 \%$, and 35.1\%, respectively (Fig 1A). Figur 1B shows a comparison of survival curves

139 between two groups of patients based on their pathologically measured tumor lengths: larger or

140 less and equal to $3 \mathrm{~cm}$. Patients who had larger tumor length showed a lower survival curve than

141 the patients with smaller tumor length. Specifically, the 3-year and 5-year OS were 44.7\% and

$14226.4 \%$ for the group with larger tumor length $(>3 \mathrm{~cm})$; whereas they were $73.1 \%$ and $49.3 \%$ for

143 the group with smaller tumor length $(\leq 3 \mathrm{~cm})$. This difference is significant $(p<0.001))$.

Univariate Coxph regression model showed that the following variables had no statistical association with survival: age, ABO blood group, family history of first degree relatives with cancer, types of esophageal resection, histologic grade, length of smoking time and length of alcohol drinking time (either as dummy variable or continuous variable). However, $\mathrm{T}$ status and

$\mathrm{N}$ status, either as dummy variable or continuous variable, and Sex, tumor length influenced significantly the OS (Table 2).

Based on the results from Univariate Coxph regression, a multivariate Coxph regression model that except Sex, the rest three variables have significant association with survival. Therefore, $\mathrm{T}$ status and $\mathrm{N}$ status (either as dummy variable or continuous variable), and tumor length 
155

156

157

158

159

160

161

162

remained as independent prognostic factors.

Based on the multivariate Coxph regression model, patients with larger tumor length $(>3 \mathrm{~cm})$ had an increased risk of death compared with patients with smaller tumor length $(\leq 3 \mathrm{~cm})$ after controlling $\mathrm{T}$ status and $\mathrm{N}$ status. The hazard ratios were 1.52 (95\% CI: 1.06-2.22) when treating $\mathrm{T}$ and $\mathrm{N}$ status as dummy variables and 1.53 (95\% CI: $1.05-2.21)$ when treating $\mathrm{T}$ and $\mathrm{N}$ status as continuous variables. When $\mathrm{T}$ status and $\mathrm{N}$ status were treated as continuous variables, the hazard ratios become 1.60 (95\% CI: 1.21-2.13) when T status increased by 1 degree after controlling $\mathrm{N}$ status and tumor length, and 1.37 (95\% CI: 1.18-1.60) when $\mathrm{N}$ status increased by 1 degree after controlling $\mathrm{T}$ status and tumor length (Table 3).

Figure 2 showed the fitted results of multivariate Coxph regression model. The top four figures showed the variation of T-status (incresing tumor depth) while holding $\mathrm{N}$-status constant at $\mathrm{N}_{0}$; the bottom four figures showed the variation of $\mathrm{N}$-satus (increasing lymph node involvement) while keeping $\mathrm{T}$-satus constant at $\mathrm{T}_{3}$. Each figure showed two curves with the top one (blue) corresponding to smaller tumor length and the low curve (green) to larger tumor length. These choices of $\mathrm{N}_{0}$ and $\mathrm{T}_{3}$ are based on the fact that most patients in the study were either in $\mathrm{T}_{3}$ (63.1\%) status or in $\mathrm{N}_{0}(59 \%)$ status (Table 1). We used the multivariable Coxph regression model to fit the survival trend of patients with $\mathrm{T}_{3} \mathrm{~N}_{0-3}$ status and $\mathrm{T}_{1-4} \mathrm{~N}_{0}$ status in the two different tumor length groups (tumor length $\leq 3 \mathrm{~cm}$ versus $>3 \mathrm{~cm}$ ). The results showed that the OS rate was higher in the smaller tumor length $(\leq 3 \mathrm{~cm})$ group independent of the tumor stage. The OS rate declined as either T-status or $\mathrm{N}$-status increases independent of the tumor length. 
176

177

178

179

180

181

182

183

184

185

186

187

\section{Discussion}

Our study showed that patients with larger tumor length than $3 \mathrm{~cm}$ had a higher risk for death than those with smaller tumor length. This indicated that tumor length, in additional to the depth of the tumor and lymph node involvement, is also an independent prognostic factors to ESCC. This finding can be important to the improvement of ESCC prognosis, giving the current poor situation on prognosis of ESCC. In fact, the results in ESCC are inconsistent so far. The worldwide studies have been trying to identify more accurate methods to determine prognosis in patients. This study provides the needed evidence for the prognostic factors to improve the ESCC.

The association between tumor length and OS rate has been controversial. Two studies conducted in Japan and one study in Germany showed that tumor length was associated with survival rate of ESCC patients in univariate analyses, but not in the multivariate analyses (Bollschweiler et al. 2006; Tachibana et al. 2002; Tachibana et al. 1999). In these studies, some patients received neoadjuvant therapy, which may have biased the measurement of tumor length because of tumor regression. Another study conducted in Japan also showed that tumor length was not an independent prognostic factor to OS of ESCC patients (Igaki et al. 2001). However, the study sample was small only 96 ESCC cases. In addition, the tumor length was measured with X-ray examination which is known to be a rough measuring method. Further more, the study did not indicate how to figure out the cut-off point. A study conducted in America showed that tumor length had no association with survival of ESCC patients (Yendamuri et al. 2009). Here again, the statistical power was small because of small sample size only 30 cases. 
197 Some studies conducted in China showed that tumor length could be as a prognostic factor in

198 ESCC (Feng et al. 2013; Ma et al. 2015; Wang et al. 2011). However, the optimum cut-off point

199 of the tumor length was different. One study in Taibei indicated that $3 \mathrm{~cm}$ was the cut-off point

200 for prediction (Wang et al. 2011); while the other study in Tianjin and the third one in Zhejiang

201 chosed $4 \mathrm{~cm}$ as the cut-off point (Feng et al. 2013; Ma et al. 2015). A large study conducted in

202 the U.S. showed that tumor length was an independent predictor of mortality and a 3-cm tumor

203 length cut-off was proposed. This agrees with our result using regression tree for survival and

204 time-dependent ROC curve methods, and multivariate Coxph regression.

205 Our study indicated that $\mathrm{T}$ classification and $\mathrm{N}$ classification both were independent prognostic

206 factors in EC. This is consistent with the known results that are acknowledged in the current

207 TNM classification (Peyre et al. 2008; Wijnhoven et al. 2007). Figure 3 showed the dependence

208 of Beta for T, N, and tumor length on time. Beta was the scaled Schoenfeld residuals from tests

209 and graphical diagnostics for proportional hazards. If the proportional hazards assumption is true,

210 beta(t) will be a horizontal line centering around 0. Since Beta for each chosen variable was

211 independent of time and basically zero, we concluded that each prognostic factor and the whole

212 model did not violate the proportional hazards assumption (S1 Table). Our results showed that

213 the survival rate decreased as either tumor depth or lymph node involvement increased after

214 controlling the other variables. The multivariable Coxph regression model indicated that tumor

215 length was positively associated with survival after adjusting the $\mathrm{T}$ and $\mathrm{N}$ status. Therefore, the

optimal cut-off point of tumor length applied to different $\mathrm{T}$ or $\mathrm{N}$ status. 
217 Studies have proven that smoking and alcohol intake increased the risk of ESCC (Chung et al.

218 2010; Islami et al. 2011), and positive synergistic effect of alcohol and tobacco use for ESCC

219 existed (Prabhu et al. 2014). Our study explored whether smoking and alcohol intake before

220 diagnosis had influence on ESCC survival. However, the study showed that alcohol and tobacco

221 intake had no association with survival. The data on smoking and alcohol were consumption

222 duration not the intensity of tobacco and the amount of alcohol usage, which was an important

223 limitation. One study showed that heavy smoking is strongly associated with poor prognosis

224 (Shitara et al. 2010).

225 In our study, we did not find the association between ESCC and either family history of any

226 cancer in first-degree relatives or family history of EC in first-degree relatives. Very few studies

227 explore the prognostic value of family history of cancer to ESCC. Other studies have shown that

228 family history of any cancer in first-degree relatives was one of the risk factor for ESCC (Bhat et

229 al. 2015; Gao et al. 2009), and Chen et al proved that family history of EC was the risk factor of

230 ESCC (Chen et al. 2015). In addition, Jiang et al indicated that family history of EC was one of

231 the independent prognostic factors for survival of ESCC (Yuequan et al. 2010). While there was

232 no obvious evidence to confirm ESCC with positive family history was a hereditary

233 predisposition (Yuequan et al. 2010). More studies are needed to confirm this association.

234 In the study, we did not find the association between ABO blood group and survival. This is a

235 controversial area. Several studies explored the ability of ABO blood type to predict prognosis of

236 ESCC, but the results were not consistent. Wang et al showed that ABO blood group had no

237 association with EC survival (Wang et al. 2015). Qin et al indicated that blood type was not an 
238 independent prognostic factor for all ESCC patients, but was an independent prognostic factor

239 for ESCC patients with negative lymph nodes (Qin et al. 2015). Yang et al found that ABO

240 blood group was an independent prognostic factors and blood group O had a worse OS than non-

241 O blood groups (Yang et al. 2014). The conflicting results could be due to the heterogeneity of

242 study populations or limited study sizes. Although there are several hypotheses which may explain the relationships observed between $\mathrm{ABO}$ blood group and cancer, the direct biologic mechanisms underlying the association are inconclusive. Therefore, whether ABO blood group can be used to predict ESCC survival or not still needs to be confirmed.

Our study indicated that tumor length was a prognostic factor for ESCC patients. However, limitations still existed in our study. The study design was a retrospective observational study.

As known, generally the evidence level of retrospective study is relatively low compared to that of prospective study. In addition, the sample size of the study was relatively small, which introduced the relatively larger $95 \%$ CI of HR (Table 3 ). In the future, we plan to conduct a multi-center prospective study to verify and quantity the results more precisely.

\section{Conclusions}

In this retrospective study of 498 patients, we have found that tumor length was an important prognostic factor for ESCC patients without receiving neoadjuvant therapy, in addition to the tumor depth and lymph node involvement. As with lung cancer and breast cancer, tumor size 
258 identify high risk patients for postoperative therapy. Further prospective study with larger sample 259 may be needed to confirm our results.

260

\section{References}

Bhat GA, Shah IA, Rafiq R, Nabi S, Iqbal B, Lone MM, Islami F, Boffetta P, and Dar NA. 2015. Family history of cancer and the risk of squamous cell carcinoma of oesophagus: a casecontrol study in Kashmir, India. Br J Cancer 113:524-532. 10.1038/bjc.2015.218

Bollschweiler E, Baldus SE, Schroder W, Schneider PM, and Holscher AH. 2006. Staging of esophageal carcinoma: length of tumor and number of involved regional lymph nodes. Are these independent prognostic factors? J Surg Oncol 94:355-363. 10.1002/jso.20569 
279 Edge SB, and Compton CC. 2010. The American Joint Committee on Cancer: the 7th edition of

280

281

282

283

284

285

286

287

288

289

290

291

292

293

294

295

296

297

298

299

the AJCC cancer staging manual and the future of TNM. Annals of surgical oncology 17:1471-1474.

Feng JF, Huang Y, and Zhao Q. 2013. Tumor length in elderly patients with esophageal squamous cell carcinoma: is it a prognostic factor? Ups J Med Sci 118:145-152. $10.3109 / 03009734.2013 .792887$

Ferlay J, Shin HR, Bray F, Forman D, Mathers C, and Parkin DM. 2010. Estimates of worldwide burden of cancer in 2008: GLOBOCAN 2008. Int $J$ Cancer 127:2893-2917. $10.1002 / \mathrm{ijc} .25516$

Gao Y, Hu N, Han X, Giffen C, Ding T, Goldstein A, and Taylor P. 2009. Family history of cancer and risk for esophageal and gastric cancer in Shanxi, China. BMC Cancer 9:269. $10.1186 / 1471-2407-9-269$

Gaur P, Sepesi B, Hofstetter WL, Correa AM, Bhutani MS, Watson TJ, Swisher SG, Group MDAEC, University of Rochester School of M, and Dentistry Foregut G. 2011. Endoscopic esophageal tumor length: a prognostic factor for patients with esophageal cancer. Cancer 117:63-69. 10.1002/cncr.25373

Heagerty PJ, Lumley T, and Pepe MS. 2000. Time-dependent ROC curves for censored survival data and a diagnostic marker. Biometrics 56:337-344.

Hess KR. 1995. Graphical methods for assessing violations of the proportional hazards assumption in Cox regression. Stat Med 14:1707-1723.

Igaki H, Kato H, Tachimori Y, Sato H, Daiko H, and Nakanishi Y. 2001. Prognostic evaluation 
for squamous cell carcinomas of the lower thoracic esophagus treated with three-field lymph node dissection. Eur J Cardiothorac Surg 19:887-893.

Islami F, Fedirko V, Tramacere I, Bagnardi V, Jenab M, Scotti L, Rota M, Corrao G, Garavello W, Schuz J, Straif K, Negri E, Boffetta P, and La Vecchia C. 2011. Alcohol drinking and esophageal squamous cell carcinoma with focus on light-drinkers and never-smokers: a systematic review and meta-analysis. Int J Cancer 129:2473-2484. 10.1002/ijc.25885

Lin Y, Totsuka Y, He Y, Kikuchi S, Qiao Y, Ueda J, Wei W, Inoue M, and Tanaka H. 2013. Epidemiology of esophageal cancer in Japan and China. J Epidemiol 23:233-242.

Ma MQ, Yu ZT, Tang P, Jiang HJ, Zhao XJ, Zhang JG, Qu DW, Jin QW, and Zhang XZ. 2015. Is tumor length a prognostic indicator for esophageal squamous cell carcinoma? A single larger study among Chinese patients. Int J Clin Exp Pathol 8:5008-5016.

Peyre CG, Hagen JA, DeMeester SR, Altorki NK, Ancona E, Griffin SM, Holscher A, Lerut T, Law S, Rice TW, Ruol A, van Lanschot JJ, Wong J, and DeMeester TR. 2008. The number of lymph nodes removed predicts survival in esophageal cancer: an international study on the impact of extent of surgical resection. Ann Surg 248:549-556. 10.1097/SLA.0b013e318188c474

Prabhu A, Obi KO, and Rubenstein JH. 2014. The synergistic effects of alcohol and tobacco consumption on the risk of esophageal squamous cell carcinoma: a meta-analysis. Am J Gastroenterol 109:822-827. 10.1038/ajg.2014.71

Qin J, Wu SG, Sun JY, Lin HX, He ZY, and Li Q. 2015. Effect of blood type on survival of Chinese patients with esophageal squamous cell carcinoma. Onco Targets Ther 8:947-953. 
322

323

324

325

326

327

328

329

330

331

332

333

334

335

336

337

338

339

340

341

Shitara K, Matsuo K, Hatooka S, Ura T, Takahari D, Yokota T, Abe T, Kawai H, Tajika M, Kodaira T, Shinoda M, Tajima K, Muro K, and Tanaka H. 2010. Heavy smoking history interacts with chemoradiotherapy for esophageal cancer prognosis: a retrospective study. Cancer Sci 101:1001-1006. 10.1111/j.1349-7006.2009.01466.x

Tachibana M, Dhar DK, Kinugasa S, Yoshimura H, Fujii T, Shibakita M, Ohno S, Ueda S, Kohno H, and Nagasue N. 2002. Esophageal cancer patients surviving 6 years after esophagectomy. Langenbecks Arch Surg 387:77-83. 10.1007/s00423-002-0290-1

Tachibana M, Kinugasa S, Dhar DK, Kotoh T, Shibakita M, Ohno S, Masunaga R, Kubota H, Kohno H, and Nagasue N. 1999. Prognostic factors after extended esophagectomy for squamous cell carcinoma of the thoracic esophagus. J Surg Oncol 72:88-93.

Tang WR, Chen ZJ, Lin K, Su M, and Au WW. 2015. Development of esophageal cancer in Chaoshan region, China: association with environmental, genetic and cultural factors. Int J Hyg Environ Health 218:12-18. 10.1016/j.ijheh.2014.10.004

Team RC. 2014. R: A language and environment for statistical computing. R Foundation for Statistical Computing, Vienna, Austria, 2012. ISBN 3-900051-07-0.

Wang BY, Goan YG, Hsu PK, Hsu WH, and Wu YC. 2011. Tumor length as a prognostic factor in esophageal squamous cell carcinoma. Ann Thorac Surg 91:887-893. 10.1016/j.athoracsur.2010.11.011

Wang BY, Liu CY, Lin CH, Hsu PK, Hsu WH, Wu YC, and Cheng CY. 2012. Endoscopic tumor length is an independent prognostic factor in esophageal squamous cell carcinoma. Ann 
Wang W, Liu L, Wang Z, Wei M, He Q, Ling T, Cao Z, Zhang Y, Wang Q, and Shi M. 2015. Impact of $\mathrm{ABO}$ blood group on the prognosis of patients undergoing surgery for esophageal cancer. BMC Surg 15:106. 10.1186/s12893-015-0094-1

346

348

349

350

351

352

353

354

355

356

357

358

359

360

361

Wijnhoven BP, Tran KT, Esterman A, Watson DI, and Tilanus HW. 2007. An evaluation of prognostic factors and tumor staging of resected carcinoma of the esophagus. Ann Surg 245:717-725. 10.1097/01.sla.0000251703.35919.02

Yang X, Huang Y, and Feng JF. 2014. Is there an association between ABO blood group and overall survival in patients with esophageal squamous cell carcinoma? Int J Clin Exp Med $15: 2214-2218$.

Yendamuri S, Swisher SG, Correa AM, Hofstetter W, Ajani JA, Francis A, Maru D, Mehran RJ, Rice DC, Roth JA, Walsh GL, and Vaporciyan AA. 2009. Esophageal tumor length is independently associated with long-term survival. Cancer 115:508-516. $10.1002 /$ cncr. 24062

Yuequan J, Shifeng C, and Bing Z. 2010. Prognostic factors and family history for survival of esophageal squamous cell carcinoma patients after surgery. Ann Thorac Surg 90:908-913. 10.1016/j.athoracsur.2010.05.060 
Figure 1 (on next page)

Kaplan-Meier survival curves $(\mathrm{S}(\mathrm{t}))$

(A) $\mathbf{S}(\mathbf{t})$ vs. time of after surgery with $95 \%$ confidence interval for overall ESCC patients. (B) $\mathbf{S}(\mathbf{t})$ vs. time of after surgery for ESCC patients stratified by tumor length $(3 \mathrm{~cm})$ 

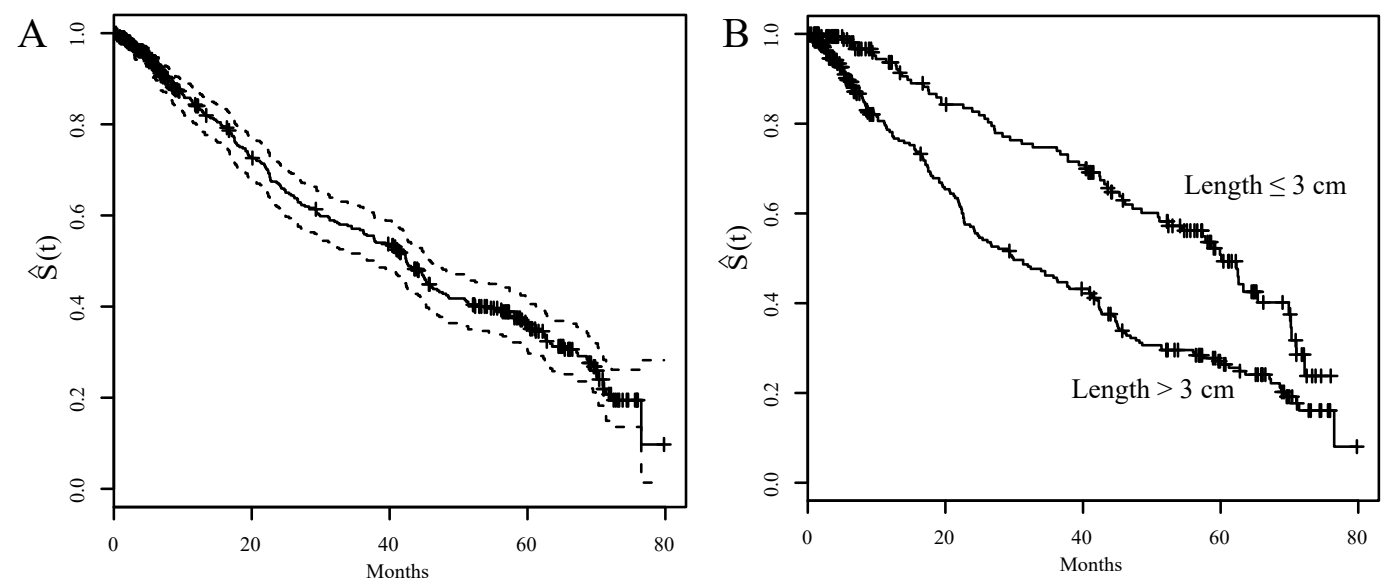


\section{Figure 2 (on next page)}

The fitted result of ESCC patients based on the multivariate Cox proportional hazard regression model

The fitted survival curve ( $\mathbf{S}(\mathbf{t})$ ) for ESCC patients with $\mathrm{T}_{1-4} \mathrm{~N}_{0}$ status (A) and $\mathrm{T}_{3} \mathrm{~N}_{0-3}$ status (B). Each figure showed $\mathbf{S}(\mathbf{t})$ for two different tumor length groups. Blue line: tumor length $\leq 3 \mathrm{~cm}$; Green line: tumor length $>3 \mathrm{~cm}$. 


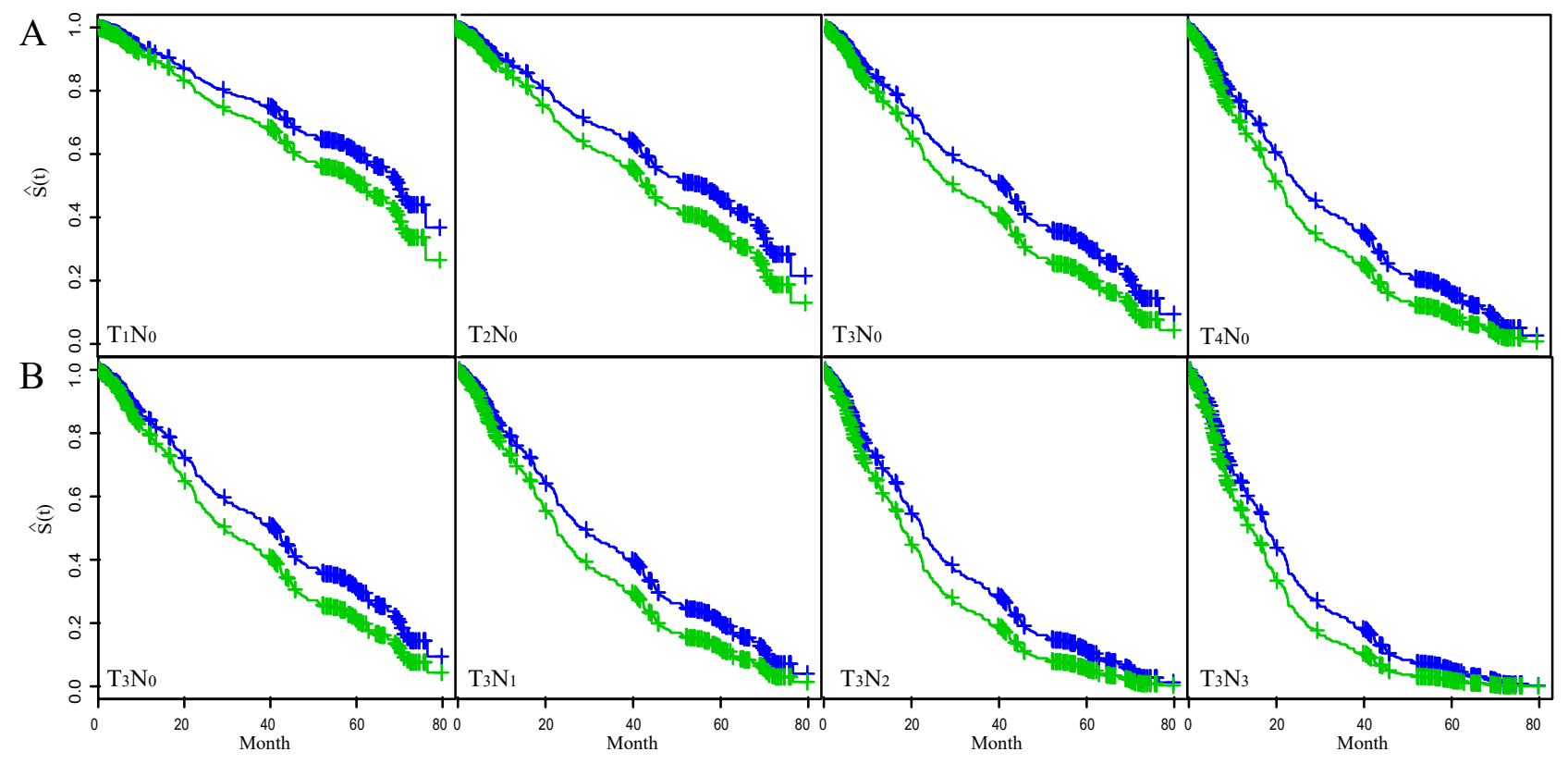


Figure 3 (on next page)

Scaled Schoenfeld residuals for each covariate.

The residual was estimated as the time-dependent coefficient beta(t) vs. transformed time. The solid line is a smoothing spline fit to the plot and the broken lines represent a \pm 2 standard-error band around the fit. The assumption of proportional hazards was supported for each of the covariates with no trend (horizontal line centering around 0 ) and p > 0.05 (S1 Table). $\mathrm{A} \square \mathrm{B}$ and $\mathrm{C}$ represent results of scaled Schoenfeld residuals against transformed time for $\mathrm{T}, \mathrm{N}$ and tumor length, respectively. 

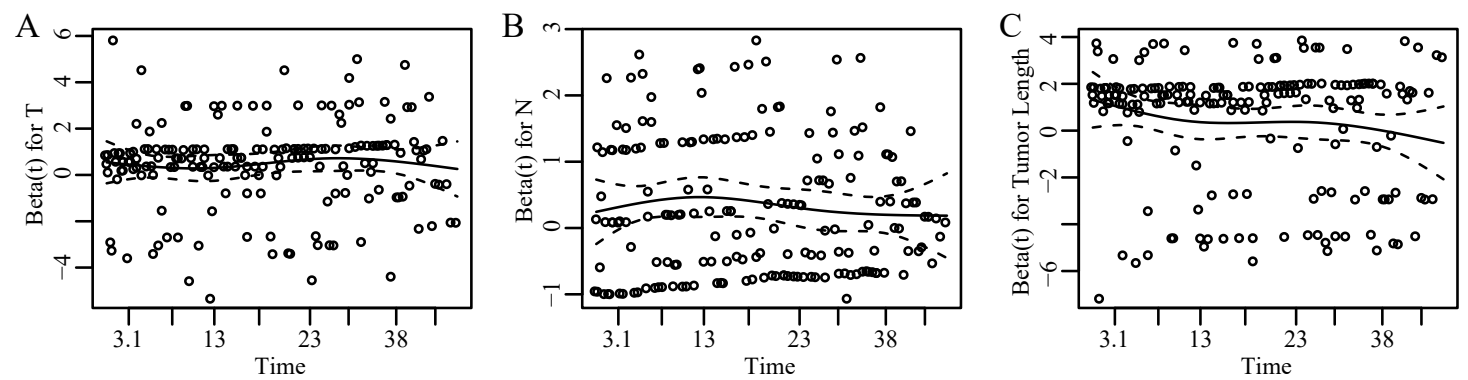
Table $\mathbf{1}$ (on next page)

Characteristics of patients 


\begin{tabular}{|c|c|}
\hline Characteristic & $(\%)$ \\
\hline \multicolumn{2}{|l|}{ Sex } \\
\hline Male & 393(78.9) \\
\hline Female & $105(21.1)$ \\
\hline \multicolumn{2}{|l|}{ Age, $y$} \\
\hline Median & 59 \\
\hline Range & $38-81$ \\
\hline \multicolumn{2}{|l|}{ ABO blood group } \\
\hline A & $152(30.5)$ \\
\hline $\mathrm{B}$ & $136(27.3)$ \\
\hline $\mathrm{O}$ & $110(22.1)$ \\
\hline $\mathrm{AB}$ & $64(12.9)$ \\
\hline Unkown & $36(7.2)$ \\
\hline \multicolumn{2}{|l|}{ Length of smoking time (year) } \\
\hline None & $157(31.5)$ \\
\hline $1-10$ & $35(7)$ \\
\hline $11-20$ & $76(15.3)$ \\
\hline $21-30$ & $130(26.1)$ \\
\hline $30-$ & $100(20.1)$ \\
\hline \multicolumn{2}{|l|}{ Length of alcohol drinking time (year) } \\
\hline None & 211(42.4) \\
\hline $1-10$ & $19(3.8)$ \\
\hline $11-20$ & $101(20.3)$ \\
\hline $21-30$ & $100(20.1)$ \\
\hline $30-$ & $67(13.5)$ \\
\hline \multicolumn{2}{|c|}{ Family history in first degree relatives with any cancer } \\
\hline None & $423(84.9)$ \\
\hline Yes & $75(15.1)$ \\
\hline \multicolumn{2}{|l|}{ Types of esophageal resection } \\
\hline Ivor-Lewis esophagectomy & $143(28.7)$ \\
\hline Three-field esophagectomy & $47(9.4)$ \\
\hline Minimally invasive esophagectomy & $17(3.4)$ \\
\hline Left thoracic & $275(55.2)$ \\
\hline Unkown & $16(3.2)$ \\
\hline \multicolumn{2}{|l|}{ Tumor length (cm) } \\
\hline$\leqslant 3$ & $185(37.1)$ \\
\hline$>3$ & $313(62.9)$ \\
\hline \multicolumn{2}{|l|}{ Tumor classification } \\
\hline Tis & $7(1.4)$ \\
\hline
\end{tabular}


Unknown

\section{Lymph node classification}

N0

294(59)

$\mathrm{N} 1$

102(20.5)

$\mathrm{N} 2$

$76(15.3)$

N3

22(4.4)

Unknown

Grade

well differentiated

$16(3.2)$

Moderately differentiated

396(79.5)

Poorly differentiated

66(13.3)

Undifferentiated

Unknown

19(3.8)

1 
Table 2 (on next page)

Univariate Cox proportional regression analyses results 


\begin{tabular}{|c|c|c|}
\hline Variable & $\begin{array}{r}\text { Univariate } \\
\text { analysis } \\
\end{array}$ & \\
\hline & $\operatorname{HR}(95 \% \mathrm{CI})$ & $p$ \\
\hline \multicolumn{3}{|l|}{ Sex } \\
\hline Male & 1.00 & \\
\hline Female & $0.64(0.43-0.94)$ & 0.023 \\
\hline Age,y & $1.00(0.98-1.02)$ & 0.836 \\
\hline \multicolumn{3}{|l|}{ Blood type } \\
\hline A & 1.00 & \\
\hline $\mathrm{B}$ & $1.42(0.83-2.44)$ & 0.198 \\
\hline $\mathrm{O}$ & $1.41(0.81-2.45)$ & 0.225 \\
\hline $\mathrm{AB}$ & $1.29(0.73-2.28)$ & 0.378 \\
\hline \multicolumn{3}{|l|}{ Length of smoking time (year) (As dummy variable) } \\
\hline None & 1.00 & \\
\hline $1-10$ & $1.03(0.57-1.86)$ & 0.910 \\
\hline $11-20$ & $0.95(0.60-1.50)$ & 0.818 \\
\hline $21-30$ & $0.98(0.66-1.44)$ & 0.906 \\
\hline $30-$ & $0.94(0.62-1.41)$ & 0.761 \\
\hline Length of smoking time (year) (As continuous variable) & $1.00(0.99-1.01)$ & 0.644 \\
\hline \multicolumn{3}{|l|}{ Length of alcohol drinking time (year) (As dummy variable) } \\
\hline None & 1.00 & \\
\hline $1-10$ & $1.35(0.65-2.80)$ & 0.424 \\
\hline $11-20$ & $1.11(0.75-1.64)$ & 0.593 \\
\hline $21-30$ & $1.04(0.70-1.55)$ & 0.842 \\
\hline $30-$ & $1.26(0.81-1.95)$ & 0.303 \\
\hline Length of alcohol drinking time (year) (As continuous & & \\
\hline variable) & $1.00(0.99-1.01)$ & 0.596 \\
\hline \multicolumn{3}{|l|}{ Family history in first degree relatives with any cancer } \\
\hline None & 1.00 & \\
\hline Yes & $0.94(0.60-1.49)$ & 0.806 \\
\hline \multicolumn{3}{|l|}{ Types of esophageal resection } \\
\hline ILE, MIE, 3-field & 1.00 & \\
\hline Left thoracic & $0.81(0.60-1.09)$ & 0.162 \\
\hline \multicolumn{3}{|l|}{ Tumor length (cm) } \\
\hline$\leqslant 3$ & 1.00 & \\
\hline$>3$ & $2.35(1.68-3.27)$ & $<0.001$ \\
\hline Tumor classification (As dummy variable) & $2.14(1.69-2.75)$ & \\
\hline $\mathrm{Tis} / \mathrm{T}_{1}$ & 1.00 & \\
\hline $\mathrm{T}_{2}$ & $4.94(1.76-13.82)$ & $<0.001$ \\
\hline $\mathrm{T}_{3}$ & $8.45(3.12-22.87)$ & $<0.001$ \\
\hline
\end{tabular}


$\mathrm{T}_{4}$

Tumor classification (As continuous variable)

Lymph node classification (As dummy variable)

$\mathrm{N}_{0}$

$\mathrm{N}_{1}$

$\mathrm{N}_{2}$

$\mathrm{N}_{3}$

Lymph node classification (As continuous variable)

Grade

well differentiated

Moderately differentiated

Poorly differentiated
$17.93(5.22-61.54)<0.001$

1.00

$2.17(1.51-3.11)<0.001$

$3.26(2.23-4.76)<0.001$

$2.73(1.45-5.13)<0.001$

1.00

$1.07(0.63-1.82) \quad 1.823$

$0.33(0.08-1.46) \quad 1.455$ 


\section{Table 3(on next page)}

Multivariate Cox proportional regression analyses results 


\begin{tabular}{|c|c|c|c|c|}
\hline \multirow[t]{2}{*}{ Variable } & \multicolumn{2}{|c|}{ Dummy variable result } & \multicolumn{2}{|c|}{ Continuous variable result } \\
\hline & $\mathrm{HR}(95 \% \mathrm{CI})$ & $p$-value & $\mathrm{HR}(95 \% \mathrm{CI})$ & $p$-value \\
\hline \multicolumn{5}{|l|}{ Sex } \\
\hline Male & 1.00 & & 1.00 & \\
\hline Female & $0.74(0.49-1.10)$ & 0.138 & $0.72(0.49-1.08)$ & 0.11 \\
\hline \multicolumn{5}{|l|}{ Tumor length $(\mathrm{cm})$} \\
\hline \multicolumn{5}{|l|}{$\leqslant 3$} \\
\hline$>3$ & $1.52(1.05-2.21)$ & 0.026 & $1.53(1.06-2.22)$ & 0.025 \\
\hline Tumor classification & & & $1.60(1.21-2.13)$ & $<0.001$ \\
\hline Tis/ $\mathrm{T}_{1}$ & 1.00 & & & \\
\hline $\mathrm{T}_{2}$ & $4.02(1.43-11.33)$ & 0.009 & & \\
\hline $\mathrm{T}_{3}$ & $4.82(1.72-13.50)$ & 0.003 & & \\
\hline $\mathrm{T}_{4}$ & $7.22(1.93-27.00)$ & 0.003 & & \\
\hline Lymph node classification & & & $1.37(1.18-1.60)$ & $<0.001$ \\
\hline $\mathrm{N}_{0}$ & 1.00 & & & \\
\hline $\mathrm{N}_{1}$ & $1.67(1.16-2.42)$ & 0.006 & & \\
\hline $\mathrm{N}_{2}$ & $2.21(1.48-3.30)$ & $<0.001$ & & \\
\hline $\mathrm{N}_{3}$ & $2.14(1.13-4.07)$ & 0.02 & & \\
\hline
\end{tabular}

1 